\title{
RELIABILITY OF RATINGS OF PERCEIVED EFFORT REGULATION OF EXERCISE INTENSITY
}

\author{
R. G. ESTON and J. G. WILLIAMS, PhD
}

School of Physical Education and Recreation, University of Liverpool

\section{ABSTRACT}

This study assessed the reliability of ratings of perceived exertion (RPE) for the prescription of exercise intensity during cycling. Ten healthy men (21 to 62 years) and six women ( 25 to 50 years) attended the laboratory four times, 5 to 7 days apart. On the first visit each subject's maximal oxygen uptake was measured and, subsequently, they cycled at constant work rates based on their perception of ratings 9,13 and 17 of the Borg 6-20 scale by regulating the resistance of the ergometer without reference to the instrument display panel. Analysis of variance revealed no significant between-trial differences with regard to oxygen uptake $\left(\mathrm{VO}_{2}\right)$ or heart rate for men or women. The relative exercise intensities corresponding to the 3 ratings of exertion did not differ between men and women. Between-trial correlations for $\mathrm{VO}_{2}$ were highest for visits two and three at RPE levels 9 and $13(r=0.83$ and $r=0.94)$ and consistently high $(r=0.92$ or greater $)$ for the three trials at RPE17. These data suggest that RPE is a useful frame of reference for the regulation of high levels of exercise intensity in healthy men and women. Small amounts of practice with the scale improve its applicability at lower exercise levels.

Key words: Effort perception, Exercise intensity control, Reliability

\section{INTRODUCTION}

The rating of perceived exertion (RPE) (Borg, 1977) is an accepted method of assessing exercise intensity. It is usually applied either as a means of estimating pre-selected power outputs (response) or in producing an appropriate power output for a pre-selected RPE (production) (Myles and Maclean, 1987). The production method assumes that a person can adjust the power output to match numerically anchored verbal expressions of effort. This procedure is recommended by an authoritative advisory body (the American College of Sports Medicine, 1986) and has been used to prescribe training intensity levels in both adult fitness (Burke and Collins, 1984) and cardiac rehabilitation programmes (Gutmann et al, 1981). The production method is claimed to be easier for a person to apply than monitoring either exercise or recovery heart rate. Also, it is said to be safer because it makes no assumptions about a person's fitness level or state of health (Myles and Maclean, 1987). However, there is very little evidence to support these assertions.

Smutok et al (1980) found that healthy young men 'learned' to use the Borg 6-20 scale to regulate the intensity of treadmill running in two sessions. This finding was based on data which showed no differences in both oxygen uptake and heart rate at pre-selected steady state mean RPE levels of 12 and above between sessions. The reliability coefficients reported were $r=0.83$ or better. Eston et al (1987) observed that healthy young men and women produced similar relative exercise intensities $\left(\% \mathrm{VO}_{2}\right.$ max $)$ at pre-selected RPE levels of 9, 13, and 17 during treadmill running. The smallest variance in $\% \mathrm{VO}_{2}$ max was observed at the higher RPE levels in both groups. The reliability of the production protocol was not formally assessed but the results from the study by Eston et al and Smutok et al suggest that the repetition of effort production is reasonably consistent, particularly at the higher levels of the Borg 6-20 scale.

In order to demonstrate that the RPE is sufficiently robust and consistent for use in exercise prescription, it would be

\section{Address for correspondence:}

Dr. R. G. Eston

School of Physical Education and Recreation

University of Liverpool

PO Box 147

Liverpool L69 3BX necessary to determine trial to trial invariance with a representative sample of those who engage in exercise. Thus, it was the purpose of this study to assess the reliability of the Borg 6-20 scale as a means of regulating exercise intensity by analysing repeated trials of exercise on a cycle ergometer in healthy adult men and women.

\section{SUBJECTS AND METHODS}

Ten healthy men lage $28.7 \pm 12.5$ yrs, height $183.1 \pm 8.9 \mathrm{~cm}$,

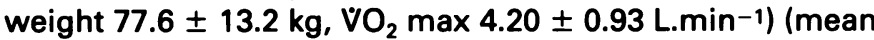
$\pm \mathrm{SD}$ ) and 6 healthy women (age $31.8 \pm 9.4 \mathrm{yrs}$, height $166.5 \pm 5.5 \mathrm{~cm}$, weight $61.2 \pm 4.9 \mathrm{~kg}, \mathrm{VO}_{2} \max 2.27 \pm$ $0.23 \mathrm{~L}$. $\mathrm{min}^{-1}$ ) volunteered to take part in this study. Subjects attended the laboratory four times. On the first visit they completed a graded exercise test on an electrically-braked cycle ergometer (Mijnhardt KEM2) to assess maximal oxygen uptake $\left(\mathrm{VO}_{2} \mathrm{max}\right)$. The protocol for this test was that recommended by Thoden et al (1982). Oxygen uptake was measured continuously using an Oxycon 4 ergoanalyser (Mijnhardt). Exercise heart rate (HR) was measured by a Lifetrace 12 Cardiometer (Albury Instruments). The Borg 6-20 RPE scale was explained to the subjects and RPE was collected in the last 15 seconds of each increment in power output using the procedure recommended by Morgan (1981). Following the first visit, a copy of the scale was given to the subjects with the request that they familiarise themselves fully with the numerical system for assessing effort and the verbal descriptors of effort which are anchored at seven levels on the scale.

On subsequent occasions, which were no less than five and no more than seven days apart, subjects were requested to cycle at constant loads based on their perception of ratings 9,13 , and 17 on the Borg scale, in that order. For each RPE the subject was asked to adjust his or her power output to an appropriate level by means of an unmarked control dial. Each was allowed three minutes to adjust power output to match the assigned RPE value. Steady state $\mathrm{VO}_{2}$ was recorded in the 4th minute after the power output was selected.

\section{RESULTS}

Tables I and II show the mean oxygen uptake and heart rate values, respectively, at each RPE level on the effort production tests $\left(T_{1}, T_{2}, T_{3}\right)$ for men and women. $A$ repeated measures analysis of variance at each RPE level revealed a 
TABLE I

A comparison of oxygen uptake (L. $\left.\min ^{-1}\right)$ between trials $\left(T_{1}, T_{2}, T_{3}\right.$ ) at each RPE lovel for men and women

\begin{tabular}{llcllll}
\hline RPE & $T_{1}$ & $\begin{array}{c}T_{2} \\
\text { Men }\end{array}$ & $T_{3}$ & $T_{1}$ & $\begin{array}{c}T_{2} \\
\text { Women }\end{array}$ & $T_{3}$ \\
\hline
\end{tabular}

$\begin{array}{lllllll}9 & 1.11(0.28) & 1.30(0.37) & 1.32(0.28) & 1.09(0.24) & 0.87(0.14) & 0.93(0.11)\end{array}$

$\begin{array}{lllllll}13 & 2.08(0.40) & 2.31(0.60) & 2.20(0.40) & 1.47(0.12) & 1.34(0.08) & 1.37(0.11)\end{array}$

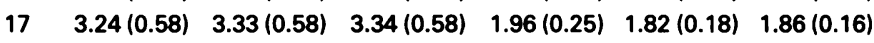

Values are the means (SD) for 10 men and 6 women.

There were no significant differences between trials, for either men or women subjects $(P>0.05)$.

TABLE ॥

A comparison of heart rate between trials $\left(T_{1}, T_{2}, T_{3}\right)$ at each RPE level for men and women

\begin{tabular}{rcccccc}
\hline RPE & $T_{1}$ & $\begin{array}{c}T_{2} \\
\text { Men }\end{array}$ & $T_{3}$ & $T_{1}$ & $\begin{array}{r}T_{2} \\
\text { Women }\end{array}$ & $T_{3}$ \\
\hline 9 & $87(9.7)$ & $94(19.3)$ & $94(13.5)$ & $117(14.2)$ & $107(2.9)$ & $109(13.9)$ \\
13 & $116(12.0)$ & $122(17.9)$ & $120(11.0)$ & $144(11.5)$ & $137(8.2)$ & $134(17.1)$ \\
17 & $150(13.7)$ & $154(13.4)$ & $152(10.3)$ & $158(12.3)$ & $155(7.3)$ & $154(11.5)$
\end{tabular}

Values are the means (SD) for 10 men and 6 women.

There were no significant differences between trials, for either men or women subjects $(P>0.05)$.

non-significant value $(P>0.05)$. Independent t-tests showed that there were no differences between men and women for self-selected relative exercise intensity at any of the 3 RPE levels examined. As both groups produced a similar $\% \mathrm{VO}_{2}$ max at each RPE level, the data were pooled and a correlational analysis of absolute $\mathrm{VO}_{2}$ and $\mathrm{HR}$ levels between trials was computed. Correlation values were highest for the last two trials $\left(T_{2}\right.$ and $\left.T_{3}\right)$ at RPE9 and RPE13 and consistently high between all three trials at RPE17. These data are listed in Table III.

TABLE III

Correlation between trials $\left(T_{1}, T_{2}\right.$ and $\left.T_{3}\right)$ for $\mathrm{VO}_{2}$ and $H R$ at each RPE level

\begin{tabular}{rrrrrrr}
\hline RPE & $\mathrm{T}_{1}-\mathrm{T}_{2}$ & $\begin{array}{c}\mathrm{VO}_{2} \\
\mathrm{~T}_{1}-\mathrm{T}_{3}\end{array}$ & $\mathrm{~T}_{2}-\mathrm{T}_{3}$ & $\mathrm{~T}_{1}-\mathrm{T}_{2}$ & $\begin{array}{c}\mathrm{HR} \\
\mathrm{T}_{1}-\mathrm{T}_{3}\end{array}$ & $\mathrm{~T}_{2}-\mathrm{T}_{3}$ \\
\hline 9 & 0.26 & 0.14 & 0.83 & 0.46 & 0.47 & 0.77 \\
13 & 0.64 & 0.72 & 0.94 & 0.53 & 0.54 & 0.75 \\
17 & 0.92 & 0.97 & 0.96 & 0.70 & 0.77 & 0.71 \\
\hline
\end{tabular}

$r_{c v(15 d f) .01}=0.61$

\section{DISCUSSION}

Overall, the results provide some support for the view that the Borg 6-20 scale is a useful device for gauging intense effort in cycling when used by healthy adult men and women. The correlation coefficients in Table III demonstrate a strong trend towards greater reliability both as the work becomes more intense and with more experience on the task (with the exception of the high level of intensity). These results hold for both the heart rate and oxygen uptake measures. This finding is in accord with results reported by Smutok et al (1980) who concluded that, for running, exercise prescription mediated by RPE provides a safe and reliable heart rate response above $80 \%$ HR max (RPE 12 and above). In the present study, an additional trial was administered and this resulted in relatively high correlations between RPE and both HR and $\mathrm{VO}_{2}$, even at the lower RPE levels. This finding suggests that further practice could extend the use of the scale to lower exercise intensities, at least for cycling.

The reliability of heart rate measures as well as $\mathrm{VO}_{2}$ was included in the study becaue of the frequent use of the former in exercise facilities and clinical settings whereas analysis of oxygen uptake would be the more critical measure recorded in the laboratory setting. It was noteworthy that the correlations between RPE and heart rate were lower at all levels of the analysis than those for $\mathrm{VO}_{2}$, leaving some $40 \%$ of the variance unaccounted for overall. The reason for this is difficult to ascertain, however, heart rate is known to fluctuate on an intra- and inter-subject basis as a result of environmental and emotional influences and thus may become a confounding variable in studies of this kind. Whereas oxygen uptake provides more fundamental assessment of energy cost and may be less susceptible to change during similar and repeated perceived levels of work on a cycle ergometer.

For RPE to be useful in exercise prescription, its reliability needs to be demonstrated for different types and levels of exercise. In this context Pandolf (1983) has stated that if exercise prescription is to be based on RPE then exercise mode must be specified because the source of the effort percept varies and influences the magnitude of the rating. Bearing this in mind, we carried out a supplementary analysis by comparing data from the present study with that of prior study of treadmill running in which both the protocol and subjects were similar (Eston et al, 1987). Although there are inherent difficulties with such comparisons, we believe the results, which are set out in Table IV, to be generally informative in that they support the view advanced by Pandolf. These show that at each level of RPE, \% $\% \mathrm{VO}_{2}$ max values were significantly lower $(P<0.01)$ for cycling than for running.

\section{TABLE IV}

A comparison of percentage of maximal oxygen uptake elicited at equivalent RPE levels during cycling and running

\begin{tabular}{rcccc}
\hline & Treadmill Data & \multicolumn{4}{c}{ Cycle Ergometry } \\
RPE & $n=28$ & $n=16$ & $t$ & $p$ \\
\hline 9 & $48.8(19.2)$ & $35.5(10.2)$ & 4.16 & $<0.001$ \\
13 & $70.4(6.5)$ & $56.9(9.3)$ & 5.65 & $<0.001$ \\
17 & 89.0 & $81.5(9.5)$ & 3.34 & $<0.01$ \\
\hline
\end{tabular}

Values are the means (SD) for 16 men and 12 women (treadmill) and 10 men and 6 women (cycle ergometer)

In summary, the results reported here support the proposition that the RPE can be used as a reliable frame of reference for the production of appropriate levels of effort by healthy adults during cycle ergometry. The recommendation of the American College of Sports Medicine (1986) which suggests the prescription of an exercise intensity of between 12 and 16 on the Borg scale has qualified support from the findings of this study. The qualifying condition being that habitually sedentary individuals would not exercise at the higher end of the 12 to 16 range. The mean exercise intensity attained at RPE17 by healthy subjects in this and our previous study was $89 \% \mathrm{VO}_{2}$ max for running and $81 \% \mathrm{VO}_{2}$ max for cycling. Clearly, such levels would not 
be recommended for individuals who were judged to be in a 'high-risk' category.

Finally, although our subjects were requested to produce selected levels of effort on only three consecutive occasions, there was a strong trend towards improved correspondence of RPE and both heart rate and $\mathrm{VO}_{2}$ at the lower levels of RPE. This suggests that practice is required if RPE is to be employed with confidence as a prescriptive device where most judgements are likely to be made in the intermediate effort levels of the scale.

\section{References}

American College of Sports Medicine, 1986. Guidelines for graded exercise testing and prescription. Philadelphia, Lea and Febiger.

Borg, G. A. V., 1977. Physical work and effort. Oxford, Pergamon Press.

Burke, E. J. and Collins, M. S., 1984 "Using perceived exertion for the prescription of exercise in healthy adults". In: Clinical Sports Medicine (Ed.) R. C. Cantu, Lexington, Mass., Collamore Press pp. 93-105.
Eston, R. G., Davies, B. L. and Williams, J. G., 1987 "Use of perceived effort ratings to control exercise intensity in young healthy adults". European Journal of Applied Physiology 56: 222-224.

Gutmann, M. C., Squires, M. L., Pollock, M. L., Foster, C. and Anholm, J., 1981 "Perceived exertion - heart rate relationship during exercise testing and training in cardiac patients". Journal of Cardiac Rehabilitation 1: 52-59.

Morgan, W. P., 1981 "Psychophysiology of self-awareness during vigorous physical activity". Research Quarterly for Exercise and Sport 52: 385-427.

Myles, W. S. and Maclean, D., 1987 "A comparison of response and production protocols for assessing perceived exertion". European Journal of Applied Physiology 55: 585-587.

Pandolf, K. B., 1983 "Advances in the study and application of perceived exertion". In: Exercise and Sports Science Reviews (Ed.) R. Terjung, New York, Pergamon Press pp. 118-158.

Smutok, M. A., Skrinar, G. S. and Pandolf, K. B., 1980 "Exercise intensity: subjective regulation by perceived exertion". Archives of Physiology, Medicine and Rehabilitation 61: 569-574.

Thoden, J. S., Wilson, B. A. and MacDougall, J. D., 1982 "Testing aerobic power". In: Physiological testing of the elite athlete (Eds.) J. D. MacDougall, H. A. Wenger, H. J. Green, Winsor, Ontario, Canadian Association of Sports Sciences pp. 39-60.

Title:

\section{BOOK REVIEW}

Editors: $\quad$ A. Dirix, H. G. Knuttgen and K. Tittel

Publisher: $\quad$ Blackwell Scientific Publications, Oxford, 1988

\begin{tabular}{|c|c|c|c|c|}
\hline Price: $£ 39.50$ & 692 pages & Numerous figures & Index & ISBN 0632019638 \\
\hline
\end{tabular}

Sixty-two contributors have combined to produce a most comprehensive book on sports medicine for Olympic and other national or international teams, and authors have been selected from among those prominent in the Olympic medical hierarchy and in the International Federation of Sports Medicine, "FIMS". Two of the contributors are from the UK, Peter Sperryn and Arnold Beckett, and the others, including several overseas members of BASM from all over the world. The preface is written by His Excellency J. A. Samaranch, President of the International Olympic Committee, who describes the relationship between the IOC and its Medical Commission under the chairmanship of Prince Alexandre de Merode. Many of the present medical problems facing top level sport relate to doping, - those doping "might not in fact contain the seeds of their own destruction", and this was written a few months before the 1988 Olympic Games took place, with the associated doping scandals. Another pertinent point he puts forward is the danger of the wealthy industrialised countries letting their sports science and medicine produce a sporting elite, whereas less developed countries who may have naturally gifted athletes can only use their resources merely to encourage hygiene and advise about nutrition. Further details of FIMS and its Medical Commission are given by the editors and by Prince de Merode. FIMS is "one of the largest medical societies in the world" (and BASM have made significant contributions to it over the past fifty or so years through Lord Porritt, John G. P. Williams, its past Secretary General, and now Peter Sperryn). The Medical Commission was founded in 1966, mainly to combat the unfair philosophy of "running at all costs", so from the first the fight against doping was its prominent interest, with 27 accredited laboratories world-wide for dope control. Other Sub-Commissions of FIMS Medical Commission are concerned with Physiology associated with Biomechanics; Injury; Co-ordination with National Olympic Committees including Education.

The remaining twelve parts of the book are devoted to: Physiology; Fitness Assessment; Environment (all sections, hot and cold, altitude, pollution - written by Roy Shephard); Training; Anthropometry; the Young Athlete; the Female Athlete; the Older Athlete; Sports Injuries; Exercise and Chronic Disease; Team Care; Doping. Some of the criteria laid down for team care, such as an extensive medical examination every three years, are perhaps advisable for the Olympic athlete, but impractical for every club standard athlete. The criteria for a team's accommodation and the required medical facilities are most useful, together with advice on legitimate medication to avoid adverse dope tests.

The book is very well presented, with abundant figures and graphs. Most of the colour photographs are of healthy athletes performing their chosen activity or undergoing ergometer investigations, but a few injuries are well illustrated. Each part of the book is introduced by a somewhat abstract coloured illustration of sport in action, based upon classical Greek figures. The print is clear, well spaced out and easy to read. I found few mistakes (though in nearly 700 pages and hundreds of references some could easily be missed; e.g. on page 363 "Kristoff" is mentioned, but in the appropriate reference section, it is spelt as "Krissoff").

This is a most enjoyable book to read as well as to study, and is very strongly recommended to any Team Doctor looking after a team in residence, as well as his supporting staff of physiotherapists, chiropodists and nurses. Much is also of relevance to team managers and games organisers. At a price of just under $£ 40, I$ consider it good value. 